\title{
Resiliência na Cadeia de Suprimentos: O Caso de Uma Empresa de Cosméticos
}

Supply Chain Resilience: The Case of a Cosmetics Company

\section{Resumo}

A busca pela eficiência em cadeias de suprimentos pode aumentar sua exposição à riscos de ruptura e para evitá-las, alguns elementos estão apontados no contexto da resiliência. $\bigcirc$ objetivo do trabalho foi identificar elementos para construir resiliência, tendo como foco a área de compras de uma indústria de cosméticos. Na parte empírica, foi realizado um estudo de caso. Os elementos que contribuem para a área de compras são comunicação, compartilhamento de informação e integração externa, usados na negociação com fornecedores. Identificou-se que ações para reduzir os riscos do modelo de negócio e variações de demanda são importantes para construção de resiliência.

Palavras-chave:

de Suprimentos. Cosméticos. Elementos. Venda

\section{Abstract}

The search for efficiency in the supply chains can increase their exposure to risks of rupture and to avoid them, some elements are pointed out in the context of resilience. The objective of this article was to identify elements to build resilience, focusing on the purchasing area of a cosmetics industry. In the empirical part, a case study was carried out. The elements that contribute to the purchasing area are communication, information sharing and external integration, used in negotiation with suppliers.it was identified that actions to reduce the risks of the business model and variations in demand are important for building resilience.

Keywords: 


\section{INTRODUÇÃO}

As cadeias de suprimentos enfrentam um cenário de complexidade crescente, buscando eficiência e redução de custos, busca essa que contribui para o aumento da sua vulnerabilidade. Para mitigar os efeitos das rupturas e assegurar o desempenho das cadeias, as empresas têm buscado aumentar sua resiliência (CARVALHO et al., 2012). A resiliência permite que a cadeia de suprimentos seja capaz de prever rupturas, se adaptar e responder a elas, se recuperando o mais rápido possível (BLACKHURST; DUNN; CRAIGHEAD, 2011). Para isso, é essencial que empresas desenvolvam características alinhadas com seus parceiros na cadeia para gerir tanto as mudanças necessárias, quanto as rupturas inesperadas (CHRISTOPHER; PECK, 2004; SHEFFI; RICE, 2005).

$\mathrm{O}$ interesse na resiliência é destacado em empresas que dispõem de pouca margem para atraso nas entregas e buscam uma postura mais proativa com relação à boa gestão de seus fornecedores (PEREIRA; SILVA, 2015), como é o caso da indústria de cosméticos. Estas empresas, em geral, orientam suas políticas de compra e seleção de fornecedores para economias de escala, muitas vezes adquirindo matéria-prima de um único fornecedor. Alguns desses fornecedores estão localizados em locais dispersos e distantes, o que faz com que as empresas trabalhem com lead times elevados. Observando a estratégia de distribuição de seus produtos, as empresas de cosméticos podem optar pela venda direta, que pressupõe relacionamento ágil entre revendedoras e clientes, que em geral, não comercializam apenas uma marca. Isso reforça a necessidade da garantia do nível de serviço, sob o risco de redução de sua base de revendedoras e perda de oportunidades de venda. Nesse sentido, a área de compras precisa garantir disponibilidade de matéria-prima para evitar atrasos na produção e entrega. Procurando responder a esse desafio, mantendo atenção na variável custo e seu impacto nos resultados da empresa, elevar o nível de serviço prestado às revendedoras e aumentar a satisfação dos clientes, o objetivo desse trabalho foi identificar elementos para construir resiliência, tendo como foco do estudo a área de compras de uma indústria de cosméticos.

\section{METODOLOGIA}

Esse trabalho possui natureza qualitativa e caráter exploratório, uma vez que se trata de um tema em estágio inicial de desenvolvimento (YIN, 2015), no qual proporciona a compreensão de um problema ou fenômeno pouco estudado, principalmente em um país em desenvolvimento (TUKAMUHABWA et al., 2017). Esse trabalho se iniciou com uma revisão sistemática da literatura (RSL), seguindo as diretrizes determinadas por Denyer e Tranfield (2009) foram usadas para conduzir a RSL baseada na questão: “Quais são os elementos que contribuem para a resiliência na cadeia de suprimentos?” O primeiro passo da RSL foi elaborar um protocolo, visando descrever as atividades realizadas (Quadro 1). 
Quadro 1 - Protocolo RSL

\begin{tabular}{|c|c|c|}
\hline Passo & Entrega & Informações \\
\hline $\begin{array}{c}\text { 1- Planejar revisão } \\
\text { sistemática de literatura }\end{array}$ & $\begin{array}{c}\text { Identificar estratégia } \\
\text { de estudo }\end{array}$ & $\begin{array}{l}\text { 1. Identificar constructos; 2. Definir palavras-chave; 3. Desenvolver } \\
\text { strings de busca; 4. Pesquisa nas bases de dados: } \\
\text { Proquest, Web of Science, Scopus e Scielo; } 5 \text {. Pesquisa no periodo } \\
\text { de } 2002 \text { - out } 2016\end{array}$ \\
\hline \multirow{2}{*}{$\begin{array}{c}\text { 2- Conduzir a revisão } \\
\text { sistemática de literatura }\end{array}$} & Selecionar estudos & $\begin{array}{c}\text { Filtro 1: Avaliação de título, resumo e palavras-chave; filtro 2: } \\
\text { Avaliação da introdução e conclusão; - Análise da qualidade do } \\
\text { artigo e dos critérios de inclusão e exclusão }\end{array}$ \\
\hline & Coletar informações & $\begin{array}{c}- \text { Leitura completa do artigo } \\
- \text { Análise de dados } \\
\end{array}$ \\
\hline \multirow{2}{*}{$\begin{array}{l}\text { 3- Informar e disseminar } \\
\text { resultados }\end{array}$} & Analise de dados & $\begin{array}{l}\text { - Análise de conteúdo e verificação de referências cruzadas de } \\
\text { diversos conceitos, discussões. }\end{array}$ \\
\hline & Síntese de dados & $\begin{array}{l}\text { - Responder questão da revisão sistemática de literatura } \\
\text { - Levantar informações relevantes e lacunas na literatura }\end{array}$ \\
\hline
\end{tabular}

Fonte: elaborado pelos autores.

Primeiro, constructos foram estabelecidos, como supply chain, resilience e vulnerabilities. Três bases de dados online foram utilizadas, Web of Science, Scopus e Proquest forma escolhidas com base em sua confiabilidade e usabilidade (BUCHINGER; CAVALCANTI; HOUNSELL, 2014). O estudo considerou artigos publicados entre janeiro de 2002 e outubro de 2016 devido ao aumento de pesquisas ligadas ao tema a partir deste ano (PEREIRA; CHRISTOPHER; SILVA, 2014; KAMALAHMADI; PARAST, 2016).

Foram encontrados 1464 artigos. Em seguida, um processo de triagem foi usado para avaliar a relevância dos artigos restantes de acordo com critérios de inclusão ou exclusão, como alinhamento de objetivos, foco, análise, clareza e artigos avaliados por pares (DENYER; TRANFIELD, 2009). Como resultado dos filtros, foram analisados 49 artigos buscando identificar quais são os elementos de resiliência apontados na literatura. É importante destacar que as análises feitas sobre a área de compras não emergiram da sistemática, mas de uma revisão de escopo. Além disso, a análise referente aos geradores e impactos de riscos também não foi resultado da sistemática, mas emergiram do caso.

Após a revisão de literatura, um estudo empírico possibilitou a investigação do papel dos elementos de resiliência na área de compras, buscando fornecer uma visão mais abrangente do problema e explorando-o em profundidade (YIN, 2015; BARRATT; CHOI; LI, 2011). Foi realizado um estudo de caso único (YIN, 2015), que possibilitou o estudo do fenômeno em seu contexto real, a compreensão profunda da complexidade e da natureza do fenômeno e a investigação das relações de causa e efeito (FAWCETT et al., 2014). O caso único é apropriado quando este apresenta elementos decisivos para testar a teoria (YIN, 2015). Assim, a empresa A foi escolhida devido à recorrência de rupturas na cadeia de suprimentos que afetam a disponibilidade de produtos e garantia do nível de serviço no canal de distribuição escolhido (venda direta), tratando-se de um caso emblemático para o problema estudado. 
Como unidade de análise, determinou-se as atividades relacionadas a compras e abastecimento. A coleta de informações foi feita por meio de diversas fontes de evidências (dados secundários, observações e conteúdo das entrevistas) como tentativa de esclarecer o motivo de decisões tomadas, como foram implantadas e quais os resultados obtidos (YIN, 2015; BARRATT; CHOI; LI, 2011). As entrevistas foram semiestruturadas, aplicadas em julho de 2017. Os entrevistados eram responsáveis pela negociação com fornecedores, e planejadores de atendimento das necessidades dos produtos, responsáveis pelo abastecimento dos materiais indicados pelos sistemas da empresa. A preferência por dois níveis da hierarquia da empresa foi dada devido aos níveis de relacionamento diferentes com clientes externos e internos, bem como as diferenças de visão estratégica e operacional. O Quadro 2 caracteriza os entrevistados.

Quadro 2 - Perfil dos entrevistados

\begin{tabular}{|c|c|c|c|c|}
\hline Cargo Entrevistado & Código & Tempo de empresa & Tempo no cargo & Departamento \\
\hline Comprador - Global & CompGLO & 2 anos & 1 ano & Compras \\
\hline Comprador - Brasil & CompBR & 2 anos & 1 ano & Compras \\
\hline Coordenadora - Brasil & CoordBRA & 4 anos & 4 anos & Compras \\
\hline Analista - MRP & Ana1M RP & 6 anos & 5 anos & Supply Chain \\
\hline Analista - Planejamento & AnaPLAN & 16 anos & 1 ano & Supply Chain \\
\hline Analista - MRP & Ana2M RP & 11 anos & 5 anos & Supply Chain \\
\hline Coordenadora & CoordSC & 7 anos & 4 anos & Supply Chain \\
\hline
\end{tabular}

Fonte: elaborado pelos autores.

As entrevistas foram realizadas pessoalmente, gravadas e transcritas para análise com auxílio do software QDA Miner (QDA, 2017). O codebook foi construído a partir dos elementos extraídos da RSL e completado e modificado com base em informações que emergiram dos dados empíricos. Os códigos podem ser agrupados dentro de grupos, sendo neste trabalho feitos com base na sua contribuição para a ocorrência da ruptura, estes foram: geradores de risco, geradores de ruptura e impacto da ruptura.

A lógica de relacionamento entre os grupos de códigos analisados é demonstrada na Figura 1. Assim, uma vez realizada a codificação do texto, a análise das informações geradas através do software foi comparada com o conteúdo das entrevistas, das informações obtidas através de visitas na empresa e de consultas a informações disponíveis online. 
Figura 1 - Relacionamento entre os grupos de códigos

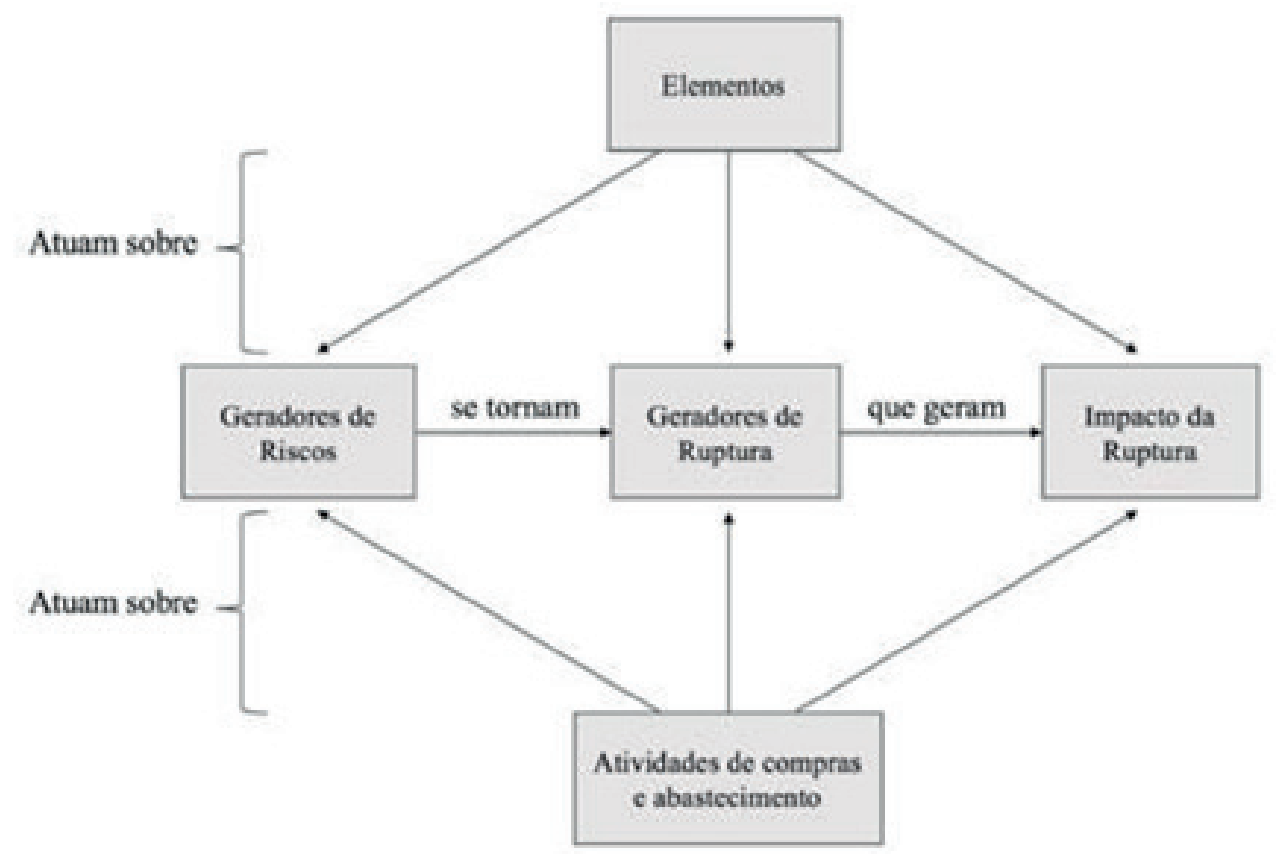

Fonte: elaborado pelos autores.

\section{REVISÃO SISTEMÁTICA DE LITERATURA}

Para fins deste estudo, resiliência é definida como a habilidade de um sistema de retornar para seu estado original ou se mover para um novo estado, mais desejável, após ser perturbado (CHRISTOPHER; PECK, 2004). Logo, apesar da impossibilidade de eliminar completamente a probabilidade de ocorrência de rupturas em sua rede de suprimentos, diversas ações podem ser tomadas para reduzir a vulnerabilidade, os riscos e elevar a capacidade de recuperação e competividade (BLACKHURST; DUNN; CRAIGHEAD, 2011). Estudos sobre a construção da resiliência envolvem o desenvolvimento do que é chamado de principles (CHRISTOPHER; PECK, 2004; KAMALAHMADI; PARAST, 2016); enablers (PEREIRA; CHRISTOPHER; SILVA, 2014), factors (AMBULKAR et al.,2015), capabilities (PETTIT et al., 2010) ou elements (ALI et al., 2017). Nesse estudo, optou- se pelo termo "elemento", definindo-o como conceito base que auxilia no desenvolvimento da resiliência. O Quadro 3 apresenta os elementos de resiliência identificados a partir da revisão sistemática de literatura e uma breve definição de cada um deles. Dentro das empresas, a área de compras é responsável pela aquisição de recursos específicos no meio externo, para a realização das atividades geradoras de valor dentro da companhia (PEREIRA et al., 2014), tendo papel fundamental na construção da resiliência, seja como ponto de contato com os elos a montante ou parte de estratégias que envolvam quaisquer dos elementos já identificados e discutidos. O objetivo da área deve ser a busca pelo alinhamento e sincronia de necessidades internas e recursos externos, para que se atinjam metas, expandindo a visão da área interna e externamente (PEREIRA et al., 2014). 
Quadro 3 - Elementos de resiliência

\begin{tabular}{|c|c|c|}
\hline Elemento & Definição & Autores \\
\hline Comunicação & \begin{tabular}{|c|} 
Capacidade de compartilhar as informações corretas e \\
estabelecer diálogo eficiente que facilite o compartilhamento \\
de todo tipo de informações, gestão de relacionamentos, \\
desenvolvimento de parcerias, tratamento de problemas e \\
colaboração.
\end{tabular} & $\begin{array}{l}\text { Christopher, Peck (2004), Stecke; } \\
\text { Kumar (2009), Blackhurst; Dunn; } \\
\text { Craighead (2011). }\end{array}$ \\
\hline Gestão de risco & $\begin{array}{c}\text { Capacidade de uma empresa identificar, coordenar esforços e } \\
\text { mitigar riscos que prejudiquem o desempenho da cadeia de } \\
\text { suprimentos, bem como o valor entregue aos stakeholders } \\
\text { envolvidos. }\end{array}$ & $\begin{array}{l}\text { Christopher; Peck (2004), Stecke; } \\
\text { Kumar (2009), Blackhurst; Dunn; } \\
\text { Craighead(2011), Tukamuhabwa } \\
\text { et al (2015). }\end{array}$ \\
\hline $\begin{array}{c}\text { Gestão de } \\
\text { relacionamento } \\
\mathrm{s}\end{array}$ & $\begin{array}{c}\text { Capacidade de gestão estratégica do tipo de relacionamento } \\
\text { desenvolvido com stakeholders. Prionizam-se empresas e } \\
\text { clientes de acordo com sua } \\
\text { importância e relevância com o objetivo de potencializar } \\
\text { vantagens competitivas e o desempenho da cadeia. }\end{array}$ & $\begin{array}{c}\text { Christopher, Lee (2004), } \\
\text { Christopher; Peck (2004), Kilubi; } \\
\text { Haasis (2015), } \\
\text { Chowdhury; Quaddus (2015), } \\
\text { Tukamuhabwa et al (2015). }\end{array}$ \\
\hline Flexibilidade & $\begin{array}{l}\text { Capacidade de uma empresa se adaptar a alterações do } \\
\text { cenário onde está inserida }\end{array}$ & $\begin{array}{l}\text { Carvalho et al (2012), Kilubi; } \\
\text { Haasis (2015), Scavarda et al } \\
\text { (2015), Chang; } \\
\text { Ellinger; Blackhurst (2015), } \\
\text { Tukamuhabwa et al (2015). }\end{array}$ \\
\hline Agilidade & $\begin{array}{l}\text { Capacidade de uma empresa se movimentar rapidamente no } \\
\text { sentido das ações que precisam ser tomadas. É facilitado por } \\
\text { processos otimizados e uma organização pouco burocrática. }\end{array}$ & $\begin{array}{l}\text { Christopher; Peck (2004), } \\
\text { Braunscheidel; Suresh (2009), } \\
\text { Speier et al (2011), Scavarda et al } \\
\text { (2015), Tukamuhabwa et al } \\
\text { (2015). }\end{array}$ \\
\hline Transparência & $\begin{array}{l}\text { Compartilhamento de informação relevantes entre os elos de } \\
\text { forma irrestrita com o potencial de aumento da confiança e } \\
\text { colaboração. }\end{array}$ & Stecke; Kumar (2009) \\
\hline $\begin{array}{c}\text { Gestão do } \\
\text { conhecimento }\end{array}$ & \begin{tabular}{|} 
Capacidade de reter, classificar e difundir o aprendizado \\
empresarial de maneira eficiente. Desenvolve-se junto com \\
de orientação para \\
aprendizado, aumentando o conhecimento e lições aprendidas \\
com cada situação. Pode ser reativo ou proativo.
\end{tabular} & $\begin{array}{l}\text { Braunscheidel; Suresh (2009), } \\
\text { Stecke; Kumar (2009), } \\
\text { Chowdhury; Quaddus (2015), } \\
\text { Tukamuhabwa et al (2015). }\end{array}$ \\
\hline Redundância & $\begin{array}{r}\text { Capacidade excedente de recursos em etapas da cadeia de } \\
\text { suprimentos capaz de suprir faltas decorrentes de choques e } \\
\text { imprevistos. Pode se dar através de fomecedores secundánios, } \\
\text { rotas e modais logisticos } \\
\text { altemativos, capacidade de manufatura excedente. }\end{array}$ & $\begin{array}{c}\text { Blackhurst; Dunn; Craighead } \\
\text { (2011), Carvalho et al (2012), } \\
\text { Chowdhury; Quaddus (2015), } \\
\text { Kilubi; Haasis (2015), } \\
\text { Tukamuhabwa et al (2015). }\end{array}$ \\
\hline Visibilidade & $\begin{array}{r}\text { Capacidade de visualizar riscos e a emergências de situações } \\
\text { atípicas que podem prejudicar ou prejudicam o desempenho } \\
\text { da cadeia de suprimentos. A visibilidade é reforçada pelo uso } \\
\text { de indicadores de desempenho (KPIs), métodos de controle } \\
\text { da qualidade e ferramentas de TI. }\end{array}$ & $\begin{array}{l}\text { Christopher; Lee (2004), Carvalho } \\
\text { et al (2012), Chang; Ellinger; } \\
\text { Blackhurst (2015), Chowdhury; } \\
\text { Quaddus (2015), Kilubi; Haasis } \\
\text { (2015), Tukamuhabwa et } \\
\text { al (2015) }\end{array}$ \\
\hline Responsividade & $\begin{array}{c}\text { Capacidade de uma empresa se posicionar e reagir com base } \\
\text { em inputs do ambiente. Englobando orientação para o } \\
\text { mercado e demanda. }\end{array}$ & $\begin{array}{l}\text { Speier et al (2011 Tukamuhabwa } \\
\text { et al (2015), Chowdhury; } \\
\text { Quaddus (2015). }\end{array}$ \\
\hline $\begin{array}{c}\text { Integração } \\
\text { interna }\end{array}$ & \begin{tabular}{|c|} 
Alinhamento e coordenação entre departamentos e \\
funcionánios da empresa, compartilhamento de informações, \\
riscos e estratégias que beneficiem a empresa como um todo \\
no sentido de maior desempenho.
\end{tabular} & $\begin{array}{l}\text { Braunscheidel; Suresh (2009), } \\
\text { Stecke; Kumar (2009). }\end{array}$ \\
\hline $\begin{array}{c}\text { Integração } \\
\text { externa }\end{array}$ & $\begin{array}{c}\text { Alinhamento e coordenação entre os elos da cadeia, } \\
\text { compartilhamento de informações, riscos e estratégias que } \\
\text { beneficiem a cadeia como um todo no sentido de maior } \\
\text { desempenho. }\end{array}$ & Braunscheidel; Suresh (2009). \\
\hline $\begin{array}{c}\text { Compartilham } \\
\text { ento de } \\
\text { informação }\end{array}$ & $\begin{array}{l}\text { Ato de compartilhar informações sobre situações atípicas } \\
\text { visualizadas na cadeia com outros elos, aumentando a } \\
\text { capacidade de resposta da cadeia } \\
\text { como um todo e facilitando ações coordena das para reduzir o } \\
\text { impacto de situaçõos emergentes e negativas na cadeia de } \\
\text { suprimentos. }\end{array}$ & $\begin{array}{c}\text { Christopher; Peck (2004), } \\
\text { Blackhurst; Dunn; Craighead } \\
\text { (2011), Speier et al } \\
\text { (2011), Kilubi; Haasis (2015), } \\
\text { Tukamuhabwa et al (2015). }\end{array}$ \\
\hline $\begin{array}{l}\text { Processos } \\
\text { otimizados }\end{array}$ & $\begin{array}{l}\text { Processos que funcionam de maneira ótima, facilitando a } \\
\text { operação e posicionamento da empresa, sem gerar custos } \\
\text { desnecessánios, retrabalho ou gargalos. Quanto mais } \\
\text { otimizados, maior a eficiência que imputam na cadeia de } \\
\text { suprimentos. }\end{array}$ & $\begin{array}{l}\text { Blackhurst; Dunn; Craighead } \\
\text { (2011), Speier et al (2011). }\end{array}$ \\
\hline $\begin{array}{c}\text { Capacidade de } \\
\text { inovação }\end{array}$ & $\begin{array}{c}\text { Capacidade de uma empresa desenvolver ou adotar soluções } \\
\text { que não foram pensadas ou implementadas anteriormente } \\
\text { para lidar comrupturas ou choques na cadeia. Traz consigo o } \\
\text { conceito de dinamismo e cultura de } \\
\text { inovaçãa. }\end{array}$ & $\begin{array}{l}\text { Blackhurst; Dunn; Craighead } \\
\text { (2011). }\end{array}$ \\
\hline
\end{tabular}

Fonte: elaborado pelos autores. 


\section{PESQUISA EMPÍRICA}

Na empresa A as atividades de compras são divididas entre dois departamentos: Compras e Supply Chain. Compras é responsável pela busca de fornecedores, negociação de custos, lead time e pela compra dos volumes necessários à produção planejada pela empresa. Supply Chain é encarregado de traçar os planos de abastecimento junto aos fornecedores segundo as variações das quantidades necessárias à produção. A cadeia é puxada e a previsão de demanda é feita pela área de Marketing, sendo refletida em necessidades de matéria prima, seguida pela negociação da área de Compras. Uma vez definidos os planos de produção, Compras entra em contato com os fornecedores e solicita a entrega das matérias primas e outros semiacabados necessários à produção. Os fornecedores entregam na planta, que processa e realiza as entregas no centro de distribuição, de onde vão para as revendedoras, utilizando muitas vezes serviços de terceiros para entregas onde sua logística não alcança.

No modelo de venda direta o contato com as revendedoras é essencial, porque traz conhecimento sobre as preferências dos clientes e a eficácia das estratégias de venda da empresa. Muitas vezes são escolhidos múltiplos fornecedores, como medida de garantia para casos de falha na entrega do fornecedor principal ou também para que variações nos volumes previstos sejam atendidas. Segundo relatos, as variações de demanda podem ser de até 400\%, gerando complicações para o abastecimento das linhas de produção. Além disso, a elevada complexidade dos produtos, que possuem dezenas de componentes em sua formulação, trava a produção pela falta de qualquer um. Para responder ao objetivo de pesquisa, a pesquisa empírica buscou entender quais são as perturbações na cadeia de suprimentos e qual a sua relação com as atividades de compras. O Quadro 4 apresenta as principais categorias avaliadas durante a revisão de escopo e aprofundadas na pesquisa empírica.

Quadro 4 - Grupos e códigos da revisão de escopo usados para análise no QDA Miner

\begin{tabular}{|c|c|}
\hline Elemento & Definição \\
\hline Geradores de risco & $\begin{array}{l}\text { Modelo de negócios, estratégia da empresa, complexidade dos produtos, contexto } \\
\text { macroeconômico, falta de alinhamento de metas e poucos fomecedores. }\end{array}$ \\
\hline $\begin{array}{l}\text { Geradores de } \\
\text { ruptura }\end{array}$ & $\begin{array}{l}\text { Falha da previsão de demanda, falha do fomecedor, falha por fatores extemos, falha na } \\
\text { manufatura, falta de matéria-prima, falha no projeto do produto e falha logística. }\end{array}$ \\
\hline mpactos & Aumento de custos, desgaste/desentendimento entre os elos e redução do nível de serviço. \\
\hline $\begin{array}{l}\text { Atividades de } \\
\text { compras e } \\
\text { abastecimento }\end{array}$ & $\begin{array}{c}\text { Escolha adequada de fomecedores, negociação de custos, negociação de lead time, procura } \\
\text { por fomecedores altemativos, prospecção de fomecedores e renegociação devido à } \\
\text { ruptura. }\end{array}$ \\
\hline
\end{tabular}

Fonte: elaborado pelos autores. 
Os geradores de riscos apresentados no Quadro 4 geram e potencializam os riscos, como riscos de processos, controle, demanda, suprimentos e ambiental (CHRISTOPHER; PECK, 2004). Os geradores de riscos se tornam geradores de rupturas (ver Quadro 4). Estas rupturas impactam no negócio e clientes, sendo prejudiciais à empresa.

Os Gráficos 1 e 2 apresentam, respectivamente a influência dos geradores de riscos e geradores de rupturas nos impactos de rupturas.

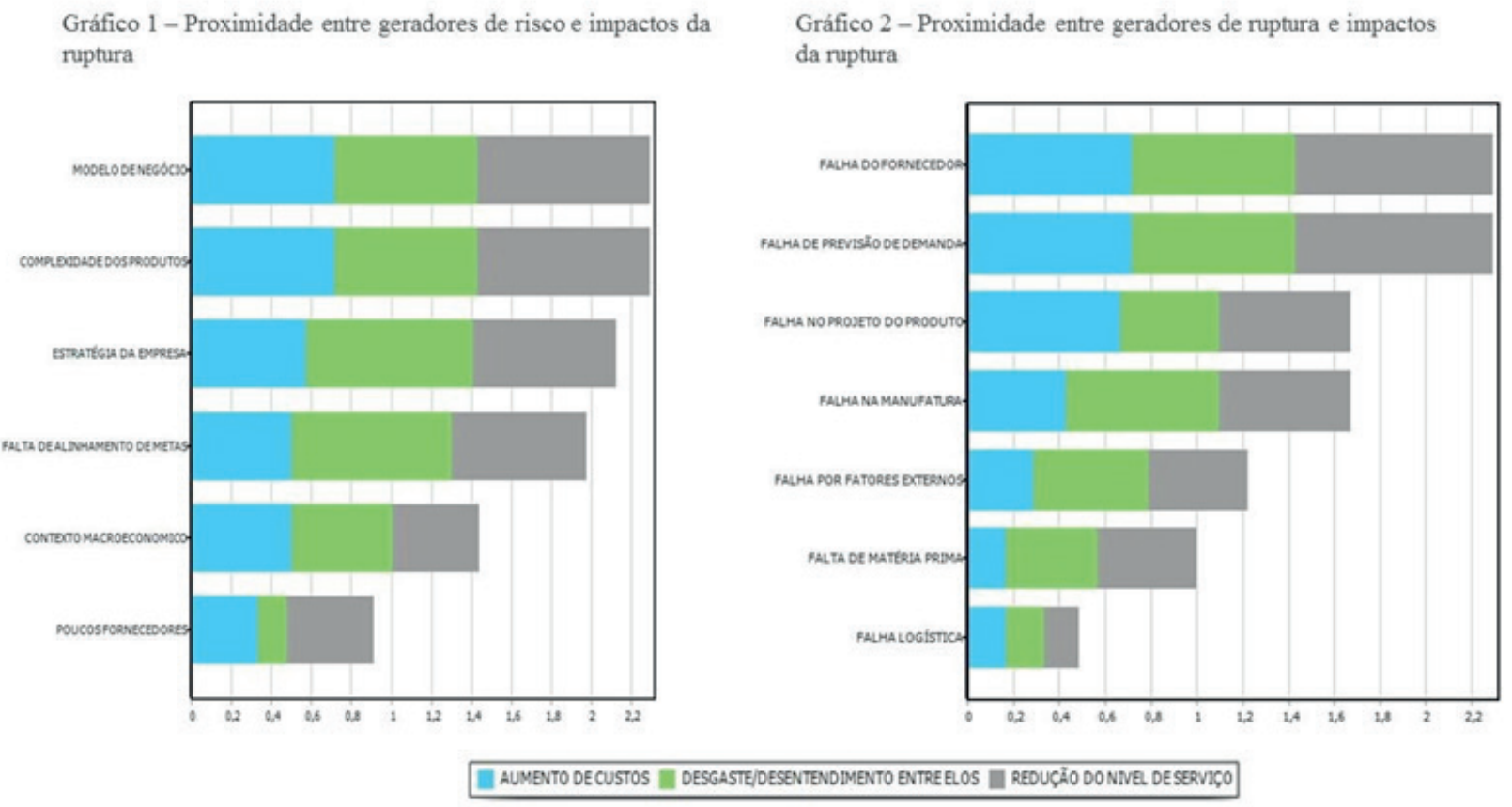

Fonte: elaborado pelos autores.

Observa-se que os riscos provenientes do modelo de negócio (venda direta), e complexidade dos produtos da empresa são os que mais impactam na empresa. Sua capacidade de penetração no mercado depende das ações das revendedoras, tornando-se naturalmente exposta a grandes incertezas vinculadas à demanda. Os entrevistados mencionam exemplos recentes de rupturas causadas pelo modelo de negócio adotado. A empresa A e suas concorrentes elaboraram modelos mistos, buscando reduzir a exposição a riscos e modernizar o acesso a seus produtos através do comércio online, mas a prática ameaça a posição das revendedoras e pode gerar conflitos. Na literatura, Christopher e Lee (2004) já destacaram esses geradores de riscos e conflitos de canal.

A complexidade dos produtos comercializados gera riscos devido ao número de matérias primas de necessárias para a formulação de cada produto. Khan, Christopher e Burnes (2008) enfatizam que a complexidade dos produtos aumenta a exposição da empresa a algumas práticas prejudiciais de alguns fornecedores, o que foi observado nas entrevistas: “(...) faltas globais na maioria das vezes nos pegam de surpresa, então quando as descobrimos já é tarde demais para conseguir reagir, e ficamos muito expostos aos preços dos fornecedores" (COORDBRA). Além disso, os processos internos da 
empresa muitas vezes não são rápidos o suficiente para se adaptar e reagir a rupturas para garantir a produção, também destacado por Ali et al. (2017). O número de fornecedores é citado como um fator de risco pelos entrevistados. Foram apontadas situações em que não existiam fornecedores suficientes no mundo para entrega de componentes no volume necessário, ou o lead time requerido para atender pedidos urgentes impossibilitava a entrega nos momentos programados.

Como mencionado, estes riscos se tornam geradores de ruptura, sendo a falha do fornecedor e a falha na previsão de demanda os que trazem maiores impactos. Estes são inter- relacionados, uma vez que a falha de planejamento leva os fornecedores a não conseguirem entregar as quantidades acordadas no prazo combinado. O processo de previsão de demanda é complexo e envolve diversos fatores, porém, a empresa possui influência limitada na sua força de vendas. Quando a demanda prevista é inferior ao número de pedidos efetivados pelas revendedoras, coloca-se em risco o nível de serviço que a empresa se compromete a entregar, enfraquecendo seu posicionamento. A partir de falhas desse tipo, a empresa inicia negociações com fornecedores principais e secundários, buscando em seguida novos fornecedores para completar as entregas. Na impossibilidade de entrega de produtos específicos para as revendedoras, a empresa envia produtos similares como forma de garantir o melhor nível de serviço possível apesar da falha. No caso de redução dos volumes demandados, buscam-se maneiras de negociar os novos volumes, ou até mesmo a permanência do estoque no fornecedor, reduzindo o custo com inventário da empresa.

Observa-se que compras atua sobre estas rupturas, uma vez que as estratégias de compras a nível global e a busca por menores custos colaboram para as metas de maior eficiência e competitividade, mas também elevam a sua exposição a riscos e rupturas que podem comprometer os ganhos inicialmente pretendidos (BLACKHURST; DUNN; CRAIGHEAD, 2011). Por outro lado, compras pode ter um papel fundamental na mitigação de riscos e, consequentemente, no aumento da resiliência (PEREIRA et al., 2014). O Gráfico 3 apresenta a relação entre os geradores de ruptura e as atividades de compras. Os principais geradores de ruptura impactados pelos processos de compras são falhas do fornecedor, da previsão de demanda e da manufatura. De acordo com o que foi identificado nas entrevistas, entende-se que as falhas mais numerosas e relevantes exigem novas negociações para que ocorra a adequação do plano de produção necessário à manutenção do nível de serviço. Exemplo disso são as mudanças significativas na demanda que forçam renegociações para a manutenção do nível de serviço; ou quando fornecedores que não são cobertos por ações de redundância e não cumprem suas entregas, sendo necessário encontrar novos outros para se manter a entrega. 
Gráfico 3 - Análise de proximidade entre atividades de compras e geradores de ruptura.

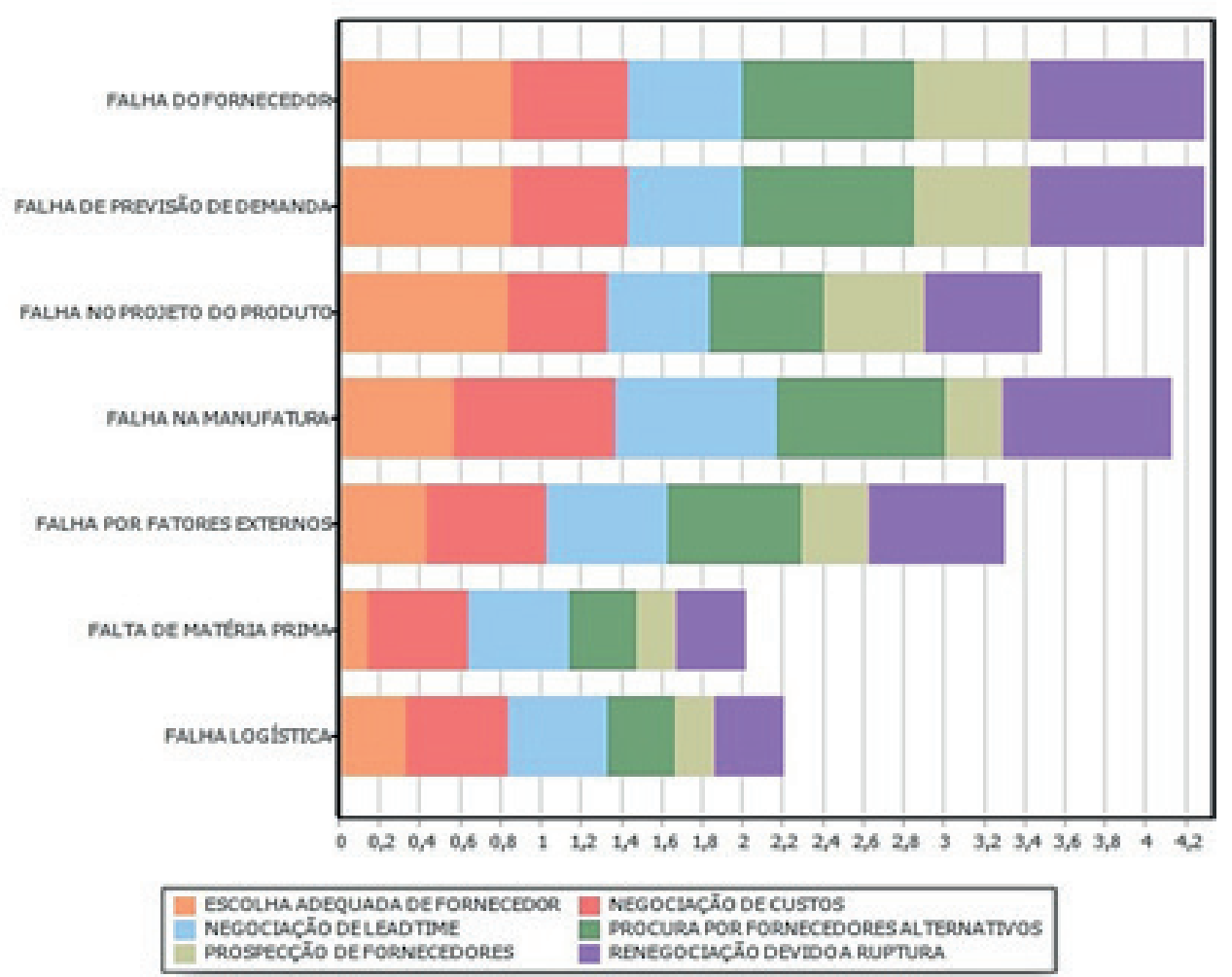

Fonte: elaborado pelos autores.

Para conseguir realizar estas ações no tempo e com estratégia adequada, Compras pode desenvolver elementos de resiliência e, assim, preparar-se melhor para lidar com as rupturas. A partir da análise do Gráfico 4, observa-se os elementos mais fortemente relacionados com as atividades de compras.

Gráfico 4 - Análise de proximidade entre elementos e atividades de compras e abastecimento.

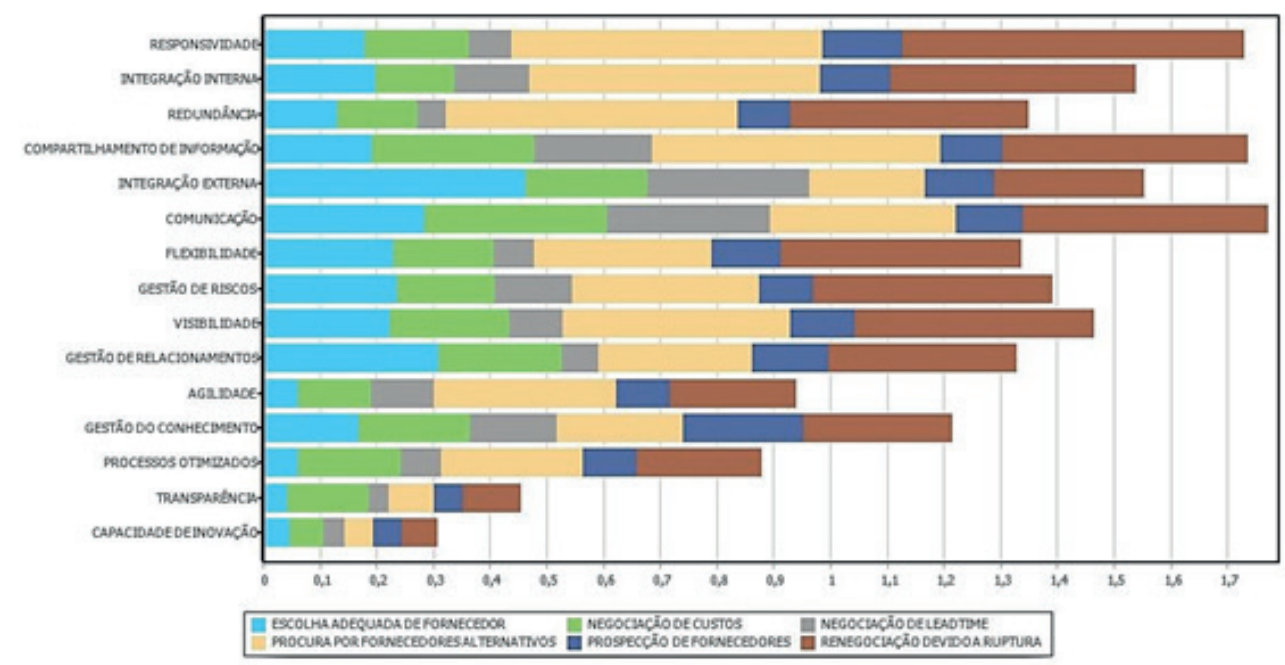

Fonte: elaborado pelos autores. 
Comunicação, compartilhamento de informação e responsividade são os elementos com maior relacionamento, dado que as principais atividades de compras destacadas exigem grande contato com os clientes, tomada de decisão e são fundamentais para o desenvolvimento de resiliência da empresa por envolverem decisões estratégicas para evitar e reagir às rupturas. Sua importância se justifica por serem o ponto de contato da empresa com seus fornecedores, executando suas estratégias de compras e abastecimento com impactos diretos no custo dos materiais e capacidade de resposta da empresa. Esses elementos

de resiliência podem mitigar os geradores de riscos e de ruptura. Por exemplo, ao fomentar maior integração entre empresa, fornecedores e revendedoras, levando a um maior entendimento entre os elos.

\section{CONSIDERAÇÕES FINAIS}

Os 15 elementos apontados na literatura foram observados durante as entrevistas, tendo destacado sua importância onde sua falta ou excesso tiveram efeitos significativos para a empresa. Redundância, por exemplo, foi destacado em todas as entrevistas como elemento relevante para se lidar com rupturas. Os elementos que mais se destacaram foram comunicação, compartilhamento de informação e responsividade, intimamente relacionados às atividades que envolvem contato e negociação com fornecedores. Na empresa, o principal objetivo da área de compras é garantir o mais alto nível de serviço com os menores custos possíveis. A importância do nível de serviço se justifica pelo modelo de venda direta adotado pela empresa, no qual seu contato com suas clientes depende das revendedoras, que promovem a empresa e ativamente buscam clientes para comercializar seus produtos. Assim, é fundamental a disponibilidade do produto e sua entrega no momento correto. Bons fornecedores são os que permitem que se atinjam esses objetivos, reduzindo ocorrência de atrasos e falha nas entregas. Para isso, o estudo de caso apontou como atividades críticas de Compras a escolha adequada do fornecedor, a procura por fornecedores alternativos e a renegociação devido à ruptura. Além disso, outros elementos de resiliência são usados em momentos de renegociação, como gestão de relacionamentos e integração externa, que facilitam o uso de vantagens competitivas para reduzir os impactos de rupturas e garantir nível de serviço.

O relacionamento das atividades de compras com os impactos de ruptura e geradores de risco é constante, indicando que o principal gerador de riscos é o modelo de negócio da empresa, seguido pela complexidade de seus produtos. Ambos submetem a empresa a fortes variações de demanda e justificam que a principal atividade de compras destacada pelos entrevistados é a renegociação devido a rupturas. Para superar estes desafios, uma medida a ser considerada pela administração da companhia é o uso de plataformas de venda diferentes, com o objetivo de reduzir a incerteza vinculada ao processo de previsão de demanda pela empresa, conforme proposto por Chowdhury e Quaddus 
(2015) como estratégia de resiliência. As falhas de entrega dos fornecedores são fonte de ruptura com forte impacto sobre o aumento de custos e a redução do nível de serviço. Apesar de a empresa fazer constante uso de estratégias de redundância, integração e gestão do relacionamento com seus fornecedores, o elevado número de matérias primas e semiacabados torna a gestão de riscos bastante complexa, sendo necessária a expansão desses elementos, ou a busca por alternativas que englobem toda a complexidade da cadeia de suprimentos da empresa. Para estes casos, o desenvolvimento de planos de contingência, por exemplo, pode aumentar a segurança da cadeia (CHANG; ELLINGER; BLACKHURST, 2015). Além disso, o elemento gestão do conhecimento merece destaque como oportunidade de desenvolvimento, uma vez que o relacionamento desenvolvido com fornecedores e melhores práticas para a construção e manutenção da resiliência são muitas vezes particulares e restritas a cada funcionário, representando um ponto de vulnerabilidade para a empresa que pode ser trabalhado desenvolvendo os elementos de integração interna e compartilhamento de informação, conforme sugerido por Braunscheidel e Suresh (2009) e Tukamuhabwa et al. (2015).

Finalmente, esta pesquisa sugere pesquisas semelhantes em outros setores e cenários são fundamentais para a construção de conhecimento no tema. Além disso, este estudo não analisou o relacionamento entre a cultura empresarial, os elementos e as práticas implementadas. Assim, é relevante aprofundar a compreensão da influência da cultura da empresa sobre os elementos, rupturas e a construção da resiliência, bem como a viabilidade da aplicação dessas ferramentas na empresa e sua influência sobre os elementos.

\section{Referências}

ALI, A., MAHFOUZ, A.; ARISHA, A. Analysing supply chain resilience: integrating the constructs in a concept mapping framework via a systematic literature review. Supply Chain Management: An International Journal, v.22, n.1, p.16-39, 2017.

AMBULKAR, S.; BLACKHURST, J.; GRAWE S. Firm's resilience to supply chain disruptions: Scale development and empirical examination. Journal of Operations Management, v.33-34, p.111-122, 2015.

BARRATT, M.; CHOI, T. Y.; LI, M. Qualitative case studies in operations management: Trends, research outcomes, and future research implications. Journal of Operations Management, v.29, p.329-342, 2011.

BLACKHURST, J.; DUNN, K. S.; CRAIGHEAD, C. W. An Empirically Derived Framework of Global Supply Resiliency. Journal of Business Logistics, v. 32, n.4, p. 374-391, 2011. 
BRAUNSCHEIDEL, M. J.; SURESH, N. C. The organizational antecedents of a firm's supply chain agility for risk mitigation and response. Journal of Operations Management, v.27, p. 119-140, 2009.

BUCHINGER, D., CAVALCANTI, G.A.D.S.; HOUNSELL, M.S. Mecanismos de busca acadêmica: uma análise quantitative. Revista Brasileira de Computação Aplicada, v. 6, n. 1, p. 108-120, 2014.

CARVALHO, H.; BARROSO, A. P.; MACHADO, V. H.; AZEVEDO, S.; CRUZMACHADO, V. Supply chain redesign for resilience using simulation. Computers \& Industrial Engineering, v. 62 , p. 329-341, 2012.

CHANG, W.; ELLINGER, A. E.; BLACKHURST, J. A contextual approach to supply chain risk mitigation. The International Journal of Logistics Management, v.26, n. 3, p. 642-656, 2015.

CHOWDHURY, M. H.; QUADDUS, M. A. A multiple objective optimization based QFD approach for efficient resilient strategies to mitigate supply chain vulnerabilities: The case of garment industry of Bangladesh. Omega, v. 57, p. 5-21, 2015.

CHRISTOPHER, M.; LEE, H. Mitigating supply chain risk through improved confidence. International Journal of Physical Distribution \& Logistics Management, v. 34, n 5, p. 388-396, 2004.

CHRISTOPHER, M.; PECK, H. Building the Resilient Supply Chain. International Journal of Logistics Management, v.15, n. 2, 2004.

DENYER, D.; TRANFIELD, D. Producing a Systematic Review. In: D. A. Buchanan; A. Bryman (Ed.). The SAGE Handbook of Organizational Research Methods, p.671-689, Thousands Oaks, CA: Sage Publications LTD., 2009.

FAWCETT, S. E.; WALLER, M. A.; MILLER, J. W.; SCHWIETERMAN, M. A. HAZEN, B. T.; OVERSTREET, R. E. A trail guide to publishing Success: Tips on Writing Influential Conceptual, Qualitative, and Survey Research. Journal of Business Logistics, v. 35, n. 1, p. 1-16, 2014.

KAMALAHMADI, M.; PARAST, M. M., A review of the literature on the principles of enterprise and supply chain resilience: Major findings and directions for future research. International Journal of Production Economics, v. 171, p. 116-133, 2016. 
KHAN, O.; CHRISTOPHER, M.; BURNES, B. The impact of product design on supply chain risk: a case study. International Journal of Physical Distribution \& Logistics Management, v. 38, n. 5, p. 412-432, 2008 .

KILUBI, I.; HAASIS, H. Supply chain risk management enablers - A framework development through systematic review of the literature from 2000 to 2015. International Journal of Business Science and Applied Management, v. 10, n. 1, 2015.

PEREIRA, C.; CHRISTOPHER, M.; SILVA, A. Achieving supply chain resilience: the role of procurement. Supply Chain Management: An International Journal, v. 19, n. 5/6, p. 626-642, 2014.

PEREIRA, C.; SILVA, A. Key Organizational Factors For Building Supply Chain Resilience: A Multiple Case Study Of Buyers And Suppliers. Journal of Operations and Supply Chain Management, v. 8, n. 2, p. 77-95, 2015.

PETTIT, T. J.; FIKSEL, J.; CROXTON, K. L. Ensuring Supply Chain Resilience: Development of a Conceptual Framework. Journal of Business Logistics, v.31, n.1, p.1-21, 2010.

QDA, 2009. Disponível em: https://www.provalisresearch.com/ Documents/QDAMiner32.pdf/. Acesso em: Out. 2017.

SCAVARDA, L. F.; CERYNO, P. S.; PIRES, S.; KLINGEBIEL, K. Supply Chain Resilience Analysis: A Brazilian Automotive Case. Revista de Administração de Empresas, v. 55, n. 3, p. 304-313, 2015.

SHEFFI, Y.; RICE, J. B. A Supply Chain View of Resilient Enterprise. MIT Sloan Management Review, v. 47, n. 1, p. 41-48, 2005.

SPEIER, C.; WHIPPLE, J. M.; CLOSS, D. J.; VOSS, M. D. Global supply chain design considerations: Mitigating product safety and security risks. Journal of Operations Management, v. 29, p. 21-736, 2011.

STECKE, K. E.; KUMAR, S. Sources of supply chain disruptions, factors that breed vulnerability, and mitigating strategies. Journal of Marketing Channels, v. 16, n. 3, 2009.

TUKAMUHABWA, B. R.; STEVENSON, M.; BUSBY, J.; ZORZINI, M. Supply chain resilience: 
definition, review and theoretical foundations for further study. International Journal of Production Research, v. 53, n.18, p. 5592-5623, 2015.

TUKAMUHABWA, B. R.; STEVENSON, M.; BUSBY, J.; ZORZINI, M. Supply chain resilience in a developing country context: a case study on the interconnectedness of threats, strategies and outcomes. Supply Chain Management: An International Journal, v.22, n. 6, p.486-505, 2017.

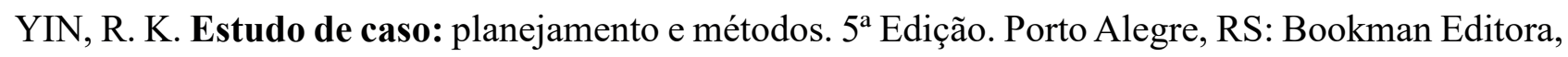
2015. 